\title{
A spectral perspective on excess volatility
}

\author{
Giacomo Livan ${ }^{\mathrm{a}, *}$, Simone Alfarano $^{\mathrm{b}}$, Mishael Milakovićc $^{\mathrm{c}}$, Enrico Scalas $^{\mathrm{d}}$ \\ ${ }^{a}$ International Centre for Theoretical Physics, Strada Costiera 11, I-34151 Trieste, Italy \\ ${ }^{b}$ Department of Economics, Universitat Jaume I, Campus del Riu Sec, E-12071 Castellón, Spain \\ ${ }^{c}$ Department of Economics, University of Bamberg, Feldkirchenstraße 21, D-96052 Bamberg, Germany \\ ${ }^{d}$ Department of Mathematics, University of Sussex, Falmer, BN1 9QH Brighton, United Kingdom
}

\begin{abstract}
We perform a rather careful spectral analysis of the correlation structures observed in real and financial returns for a large pool of long-lived US corporations, and find that financial returns are characterized by strong collective fluctuations that are absent from real returns. Once the excessive comovement is subtracted from individual financial time series, the behavior of real and financial returns is virtually identical in both the cross-sectional and time series domain, thereby demonstrating the inherently collective nature of excess volatility. Put differently, if excess volatility is to be reduced then one should probably try to inhibit excess comovement first. At any rate, the excessive behavior in volatility and comovement should not be studied in isolation of each other.
\end{abstract}

Keywords: excess volatility, excess comovement, random matrix theory, profit rate, return on assets

JEL: D22, L10, C38

\section{Introduction}

The allocation of capital faces the fundamental choice between real economic activity and financial investment. In the spirit of the efficient market hypothesis, the financial returns of a corporation should reflect the returns from its real economic activity, because sustained positive deviations between financial and real returns boil down to a Ponzi scheme, while sustained negative differences should be eliminated by arbitrage considerations along Tobin's $q$ theory of investment. From a multivariate perspective, crosscorrelations among stocks should therefore originate from cross-correlations in real activity, measured here by the return on assets (ROA). Since it is well known that stock prices exhibit excess comovement with respect to other measures of fundamental value, like earnings flows or factor model estimates, we expect a similar phenomenon in our comparison. But more importantly we will argue here that financial comovement in excess of our real benchmark is apparently the single source of excess volatility. The two major

\footnotetext{
* Corresponding author

Email addresses: glivan@ictp.it (Giacomo Livan), alfarano@eco.uji.es (Simone Alfarano), mishael.milakovic@uni-bamberg.de (Mishael Milaković), e.scalas@sussex.ac.uk (Enrico Scalas) 
methodological novelties with respect to the existing empirical literature on excess comovement are (i) the use of a reduced-form model of fundamentals (ROA) that produces estimated residuals with remarkably neat statistical properties, and (ii) the use of random matrix theory to robustly distinguish between genuine cross-correlations and noise in the spectra of real and financial correlation structures.

\section{Data and Methods}

The data for our study are taken from Thomson Reuters Datastream and consist of annual observations on the operating income $I$, total assets $A$, and market value $M$ of 475 US corporations that have been active in every year from 1980 to 2010. As a proxy for the return to real economic activity, we use the return on assets (or profit rate) of corporation $i$ in year $t$ and denote it by $p_{i t}=I_{i t} / A_{i t}$, while financial returns are computed as logarithmic differences in market value, $r_{i t}=\log M_{i t}-\log M_{i, t-1}$. This leaves us with $N=475$ different time series of length $T=30$.

The standard way of performing a correlation analysis on a set of $N$ random variables $X_{i}(i=1, \ldots, N)$ is to collect $T$ equally spaced observations in time $x_{i t}(i=1, \ldots, N ; t=1, \ldots, T)$, compute their correlation matrix and inspect its eigenvalue spectrum: large eigenvalues typically signal for the presence of strong correlations among all or some of the variables under consideration. To fix notation, let $c_{i j}$ be the Pearson coefficient between the standardized series $i$ and $j$, collected in an $N \times N$ cross-correlation matrix C. In our case, $T<N$ and therefore the rank of $\mathbf{C}$ is $T$. Principal component analysis informs us that, once normalized, $x_{i t}$ can be mapped onto a set of $T$ orthogonal principal components $e_{\ell}$ :

$$
z_{i t}=\frac{x_{i t}-\mu_{i}}{\sigma_{i}}=\sum_{\ell=1}^{T} \sqrt{\lambda_{\ell}} V_{i}^{(\ell)} e_{\ell t},
$$

where $\mu_{i}$ and $\sigma_{i}$ are the mean and standard deviation of the $N$ different time series, $\lambda_{1} \geq \lambda_{2} \geq \ldots \geq \lambda_{T} \geq 0$ denote the non-zero correlation matrix eigenvalues (given the rank of $\mathbf{C}, N-T$ eigenvalues are equal to zero), and $V_{i}^{(\ell)}$ denotes the $i$-th component of the $\ell$-th normalized eigenvector. Seeking dimensional reduction, one typically selects $L<T$ of the large eigenvalues of $\mathbf{C}$ that account for a substantial fraction of the variation in the original (normalized) series.

Most often, the choice of $L$ is rather arbitrary. Here on the other hand we rely on the Marčenko \& Pastur (1967) distribution of random matrix theory, which describes the eigenvalue density of purely random correlation matrices. Eigenvalues that are larger than the density's support accordingly identify large correlation structures and endogenize the choice of $L$. The upper bound of the Marčenko-Pastur density's support is $\lambda_{+}=(1+\sqrt{q})^{2} / q$, where $q=T / N$. Hence $L$ represents the number of eigenvalues (if any) that exceed $\lambda_{+}$. If the original time series do not exhibit significant serial correlations, the $L$ eigenvalues represent large-cluster cross-correlations among the original data. Notice, however, that in the presence of 
serial correlations one will observe eigenvalues that are larger than $\lambda_{+}$even in the case of vanishing crosscorrelations.

Since financial returns do not exhibit significant serial correlations (in line with the weak-form efficient market hypothesis), such a distinction appears superfluous at first. Yet corporate profit rates are well known to exhibit significant positive serial correlations (see, e.g., Mueller, 1977) that prevent a meaningful direct comparison of the spectra of real and financial returns. Put differently, we do not know to which extent the $L$ eigenvalues of the profit rate spectrum contain information on cross-correlation structures, or whether they are present at all. In order to disentangle cross- and time correlation effects on the $L$ eigenvalues of profit rates, we rely on the following stochastic differential equation (SDE) to describe changes in the profit rate of firm $i$

$$
\mathrm{d} p_{i t}=-B_{i} \operatorname{sign}\left(p_{i t}-m\right) \mathrm{d} t+D_{i} \mathrm{~d} W_{i t}
$$

where $B_{i}>0$ is a mean-reverting parameter, $D_{i}>0$ is an idiosyncratic innovation term, and $\mathrm{d} W_{i t}$ denotes Wiener increments. The parameter $m \in \mathbb{R}$ represents the average rate of corporate profitability and applies across all firms in the sample. ${ }^{1}$ Equation (2) has recently been introduced by Alfarano et al. (2012), based on the classical idea of capital reallocation in search of profit rate equalization. The SDE (2) turns out to provide a useful description of the statistical properties of corporate profit rates, because its stationary Laplace density coincides with the empirical density of profit rates, ${ }^{2}$ and it also accounts for the observed persistence in their autocorrelations that stems from the mean-reverting (deterministic) term of the SDE. The deterministic term accounts for the systematic tendency of competition to equalize profit rates, while the second (stochastic) term captures fluctuations in the idiosyncratic destinies of individual corporations. Therefore the latter represents the single genuine source of cross-correlation in profit rates if the reduced-form model (2) is not misspecified.

Formally speaking, equation (2) provides a natural framework for the maximum likelihood estimation of the parameters $B_{i}$ and $D_{i}$ through its associated Fokker-Planck equation that Toda (2012) has derived in closed form. After computing the maximum likelihood estimates $\hat{B}_{i}$ and $\hat{D}_{i}$, we extract the empirical Wiener increments from the discretized version of (2)

$$
\Delta \hat{W}_{i t}=\frac{1}{\hat{D}_{i}}\left(\Delta p_{i t}+\hat{B}_{i} \operatorname{sign}\left(p_{i t}-\hat{m}\right) \Delta t\right)
$$

where $\Delta t=1$ year, and $\Delta p_{i t}=p_{i t}-p_{i, t-1}$. The estimated residuals $\Delta \hat{W}$ are Gaussian, since we find that a Kolmogorov-Smirnov test indeed rejects the null hypothesis of normally distributed residuals for only 15 out of 475 time series at the $5 \%$ significance level. Moreover, the residuals do not exhibit statistically

\footnotetext{
${ }^{1}$ The average profit rate is remarkably stable over time and also reasonably similar across firms, therefore we use the mode of the unconditional pooled profit rate distribution, $\hat{m}=9.5 \%$.

${ }^{2}$ The ubiquity of Laplace distributions has also been observed in firm growth rates (see, e.g., Bottazzi \& Secchi, 2003).
} 
significant autocorrelations at the $5 \%$ level, in contrast to the original profit rate series (material available upon request). The fact that the estimated residuals are normally distributed and serially uncorrelated lends strong empirical support to the reduced-form model of corporate profitability in equation (2). The filtering procedure (3) is an important contribution of our letter because it copes with the problem of serially correlated profit rates, and therefore allows for a meaningful comparison of the spectra of real and financial returns.

\section{Results}

Let $\mathbf{M}$ denote the $N \times T$ data matrix of standardized financial returns, and let $\mathbf{W}$ denote the standardized residuals $\Delta W$. Then we can form the $N \times N$ correlation matrices

$$
\mathbf{C}_{W}=\frac{1}{T} \mathbf{W W}^{\mathrm{T}}, \quad \mathbf{C}_{M}=\frac{1}{T} \mathbf{M M}^{\mathrm{T}},
$$

and compute their spectra. Table 1 reports the normalized values of the eigenvalues exceeding the MarčenkoPastur threshold $\lambda_{+}$for both matrices, and Figure 1 illustrates the component distribution of the eigenvectors associated with the largest eigenvalues of both matrices. Notice that the top eigenvalue of $\mathbf{C}_{M}$ accounts for almost $30 \%$ of the overall variation in financial returns, and that the components of its corresponding eigenvector exclusively consist of entries with the same sign. This is not the case for $\mathbf{C}_{W}$, however, whose top eigenvalue accounts for less than $10 \%$ of the overall variation in profit residuals, and whose corresponding eigenvector components exhibit a 70/30 split between positive and negative signs.

[Result 1] The presence of a large top eigenvalue in $\mathbf{C}_{M}$ that is coupled with a corresponding eigenvector whose entries share the same sign has been firmly established in the literature (see, for instance, Laloux et al. (1999) or Livan et al. (2011) for details) and is typically interpreted as a market mode, i.e. a synchronous movement of stocks in the same direction, analogous to the notion of excess comovement in the empirical finance literature (see, e.g., Kallberg \& Pasquariello, 2008). Since $\mathbf{C}_{W}$ does not exhibit a market mode, our spectral perspective also indicates excess comovement.

[Result 2] Our spectral perspective allows us to isolate the contribution of the market mode to the time series of financial returns. Remarkably, and perhaps rather unexpectedly, it turns out that the mere removal of the largest spectral component is by itself sufficient to reconcile the correlation structure of real and financial returns. More precisely, we can construct a synthetic mean-centered time series of financial returns from equation (1) that subtracts the market mode in $\mathbf{C}_{\mathbf{M}}$ (so the summation now runs from $\ell=2$ )

$$
\tilde{x}_{i t}=\sigma_{i} \sum_{\ell=2}^{T} \sqrt{\lambda_{\ell}} V_{i}^{(\ell)} e_{\ell t}
$$

and consider its corresponding cross-correlation matrix $\tilde{\mathbf{C}}_{M}$. The third column of Table 1 illustrates that the non-random eigenvalues of $\tilde{\mathbf{C}}_{M}$ are no longer significantly different from those of $\mathbf{C}_{W}$, and since the 


\begin{tabular}{cccc} 
& $\mathbf{C}_{W}$ (profit rates) & $\mathbf{C}_{M}$ (market value) & $\tilde{\mathbf{C}}_{M}$ (synthetic market value) \\
\hline$\lambda_{1} / N$ & $9.9( \pm 1.5) \%$ & $27.4( \pm 1.7) \%$ & $12.1( \pm 1.3) \%$ \\
$\lambda_{2} / N$ & $6.8( \pm 0.8) \%$ & $9.2( \pm 1.1) \%$ & $7.9( \pm 1.0) \%$ \\
$\lambda_{3} / N$ & $5.4( \pm 0.4) \%$ & $5.9( \pm 0.8) \%$ & $6.4( \pm 0.7) \%$
\end{tabular}

Table 1: Largest (normalized) eigenvalues of the cross-correlation matrices for profit rate residuals, raw financial returns, and their synthetic counterparts. Notice that the normalized eigenvalues can be directly interpreted as the fraction of the overall sample variance contained in the corresponding principal components of equation (1). As explained in Section 2, we only report eigenvalues that exceed the Marčenko-Pastur threshold, in our case $\lambda_{+}=5.2 \%$. Standard errors at the $5 \%$ significance level are shown in parentheses and have been computed via conventional bootstrapping techniques (material available upon request), demonstrating that the eigenvalues of profit rates and synthetic financial returns are not significantly different.

components associated with the top eigenvalue of $\tilde{\mathbf{C}}_{M}$ are almost evenly split between positive and negative values, a market mode is indeed no longer present. In other words, the market mode quantifies the degree of excess financial comovement from the real benchmark.

[Result 3] Figure 2 plots annual averages across the 475 companies for financial returns, profit rates, and the synthetic financial return series from equation (5). Interestingly, the mere subtraction of the market mode is sufficient to align the magnitude of real and financial fluctuations in the time domain: upon subtraction of the leading principal component, the aggregate annual fluctuations of synthetic financial returns decrease

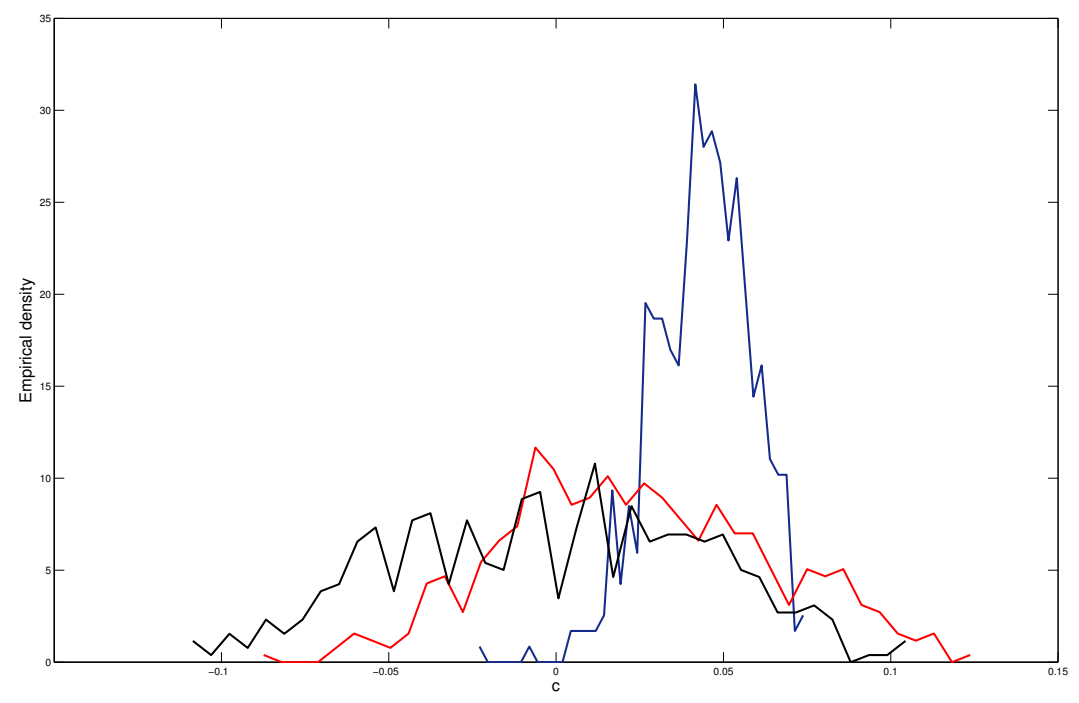

Figure 1: Empirical component distributions of the top correlation matrix eigenvectors for financial returns (solid line), profit rates (dashed), and synthetic returns from equation (5) (dotted). 
by one order of magnitude from $14.8 \%$ (standard deviation) to $1.2 \%$, comparable in magnitude to aggregate profit rate fluctuations of $1.5 \%$.

\section{Discussion and Conclusion}

Our main idea is to use profit rate cross-correlations as a benchmark in the comparison with hitherto extensively studied financial cross-correlations. The use of our reduced-form model for ROA dynamics turns out to be a very helpful device because it allows us (i) to interpret the financial market mode as a measure of excess comovement, and (ii) to show that removing the market mode makes real and financial returns consistent with each other both in the cross-sectional and the time series domain. Hence it appears that excess volatility and excess comovement are deeply intertwined. This result is not entirely trivial, as a counterexample illustrates: suppose that individual stocks exhibit excess volatility in the time domain, but that their price changes are independent of each other. Then we would observe excess volatility but not a market mode.

The more recent literature suggests various reasons for the existence of excess comovement, for instance style investing (Barberis et al., 2005), or learning from information flows regarding corporate profitability (Patton \& Verardo, 2012). While our findings are consistent with these approaches, they cannot statistically discriminate between them. On the other hand our results strongly indicate that excessive fluctuations in

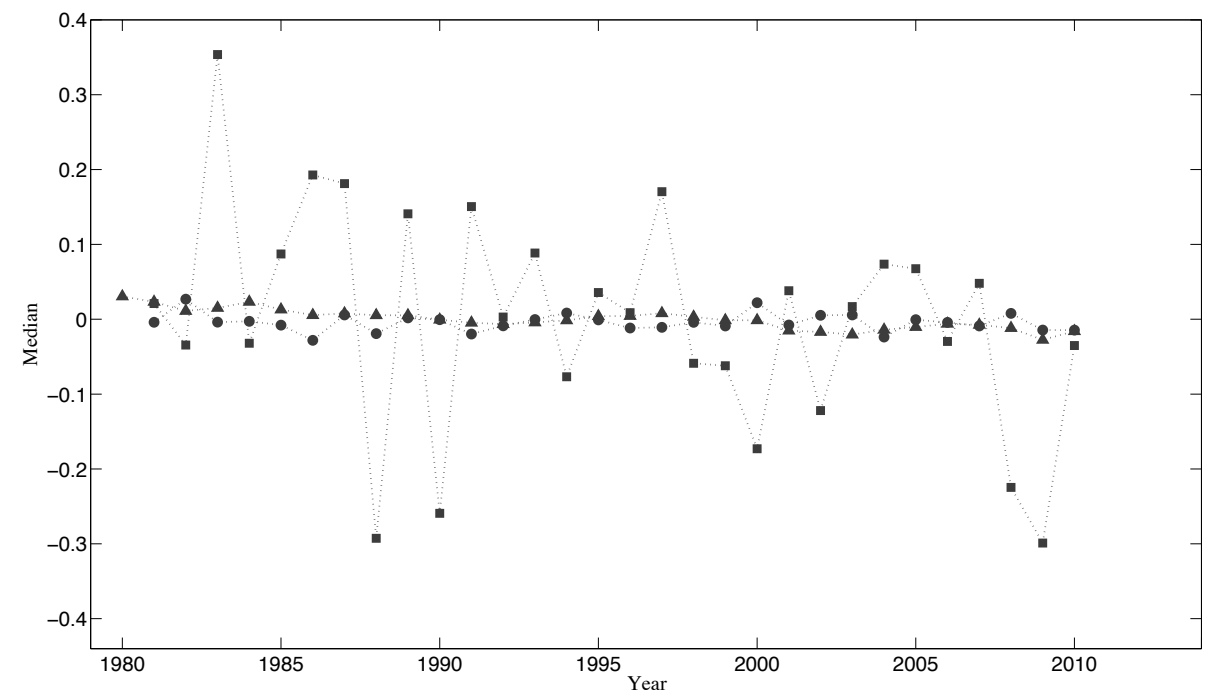

Figure 2: Annual averages computed across the 475 companies for profit rates (triangles), financial returns (squares), and synthetic financial returns (circles). Notice that synthetic returns are by construction mean-centered. Thus we have also mean-centered the other two series for visual clarity because our focus is on the magnitude of fluctuations. Concerns regarding potential differences in the levels of real and financial returns are taken up in the final section. 
the time domain (volatility) and the cross-sectional domain (comovement) should not be studied separately.

Finally, one might object that our approach suffers from a conceptual flaw in that higher financial volatility simply reflects a larger risk premium in financial investments. But the average financial rate of return in our sample is around 9.2\%, while the average profit rate is around 9.5\%, thus the Sharpe ratio of real investment dominates its financial counterpart by one order of magnitude. Incidentally, Mundt et al. (2014) corroborate that over longer horizons the real and financial rates of return are on average equal across each of the forty largest economies in the world, and that financial volatility is also an order of magnitude higher than its real counterpart. So why would capital be invested financially in the first place? From the perspective of capital owners, financial investments certainly provide considerably more liquidity than the commitment to real economic activity, and they also allow access to activities that might otherwise be outside the means of the investor (trying to build and operate, say, one millionth of a power plant is obviously a futile endeavor). Yet our results indicate that capital is paying rather dearly for liquidity and access to large scale operations in the form of excessive volatility that spans an order of magnitude.

Acknowledgements: SA thankfully acknowledges support by Ministerio de Ciencia e Innovación, project number ECO2011-23634, and the project 'Riesgo sistemico e información publica en el sector financiero: experimentos y simulaciones', financed by University Jaume I of Castellón.

Alfarano, S., Milaković, M., Irle, A., Kauschke, J., 2012. A statistical equilibrium model of competitive firms. Journal of Economic Dynamics \& Control 36, 136-149.

Barberis, N., Shleifer, A., Wurgler, J. 2005. Comovement. The Journal of Financial Economics 75, $283-317$.

Bottazzi, G., Secchi, A. 2003. Why are distributions of firm growth rates tent-shaped? Economics Letters 80, 415-420.

Kallberg, J., Pasquariello, P. 2008. Time-series and cross-sectional excess comovement in stock indexes. Journal of Empirical Finance 15, 481-502.

Laloux, L., Cizeau, P., Bouchaud, J. P., Potters, M., 1999. Noise dressing of financial correlation matrices. Physical Review Letters 83, 1467-1470.

Livan, G., Alfarano S., Scalas, E., 2011. Fine structure of spectral properties for random correlation matrices: An application to financial markets. Physical Review E 84, 016113.

Marčenko V. A., Pastur L. A., 1967. Distribution of eigenvalues for some sets of random matrices. Mathematics of the USSRSbornik 1, 457-483.

Mundt, P., Förster, N., Alfarano S., Milaković M., 2014. The real versus the financial economy: A global tale of stability versus volatility. Economics: The Open-Access, Open Assessment E-Journal 8, 2014-17.

Mueller, D., 1977. The persistence of profits above the norm. Economica 44, 369-380.

Patton, A. J., Verardo, M., 2012. Does Beta move with news? Firm-specific information flows and learning about profitability. The Review of Financial Studies 75, 2789-2839.

Toda, A. A., 2012. The double power law in income distribution: explanations and evidence. Journal of Economic Behavior \& Organization 84, 364-381. 